\title{
PARTICIPATION AND OBSERVATION IN THE MENTAL HOSPITAL
}

\author{
Robert B. Bechtel* \\ University of Kansas
}

It has been the rule that behavioral science investigators of mental hospitals describe their techniques of investigation as including, if not depending on, the method of participant observation. Yet if one adheres to the classic definition of participant observation as given by Florence Kluckhohn,

... conscious and systematic sharing, in so far as circumstances permit, in the life activities, and on occasion, in the interests and affects of a group of persons. ${ }^{1}$

it becomes clear that few investigators of the mental hospital correctly use participant observation as a technique. This fact, as it applies to many areas, has been aptly pointed out by Babchuk $^{2}$ who suggests that the observer roles listed by Gold ${ }^{3}$ adequately classify the range of situations in which observers generally find themselves doing studies, and that only one of these types is equivalent to participant observation. Gold lists four types of observer roles:

1. The Complete Participant who is the classical participant observer taking a "natural" role without others having knowledge of the intent." 2. The Participant-as-Observer who is observing persons who know about his intent.

\footnotetext{
* Robert B. Bechtel, a Social Psychologist, is Research Associate with the Program Architectural Environment and Human Behavior located at Topeka State Hospital, Topeka, Kansas. The author is indebted to James Clifton and William Stein of the University of Kansas whose criticism and stimulation made this paper possible.
}

3. The Observer-as-Participant who acts in a one-visit interview situation.

4. The Complete Observer who is entirely removed from social interactions of the persons observed.

When applied to the mental hospital setting this classification is lacking in at least two respects. One is that all of the roles chosen by observers in the past do not quite fit into these categories. Another is that the goals and techniques of observation of any kind become compromised by the hospital setting. The mental hospital is a unique subsystem ${ }^{4}$ of our society that prevents in some ways the adequate collecting of data by participant observer techniques.

Largely because of the uniqueness of the mental hospital setting and because of the special techniques it requires, a complete study of the mental hospital subsystem has not been done. Researchers have not shown they understood why the mental hospital requires techniques different from a community or an institutional study. Too often the assumption has been that we can study the mental hospital

... very much like field ethnologists who are attempting to gain knowledge about the culture of a so-called primitive group.

Perhaps some of the difficulty involved in the false assumption that one can simply transfer community observational techniques to the mental hospital lies in the fact that mental hospital observation is still a relatively recent field of 
research. So little work has been done that Levinson's observation still holds true.

We have as yet little in the way of systematic data concerning the relative advantages and disadvantages of the several types of investigator role.

The purpose of this paper will be to classify and explore the various types of roles used by past investigators under the loose category of "participant observation" and to discuss problems arising from the use of each type in relation to the unique qualities of the mental hospital subsystem.

\section{Before Assuming an Observer Role}

When the researcher decides to enter the mental hospital his social position, prior to assuming a hospital role or in assuming any but a strictly Complete Participant role, ${ }^{7}$ is somewhat antithetical to that of professional groups in the hospital setting. The psychiatrists, nurses, adjunctive therapists, and aides or attendants see the mental hospital as their own field of professional competence about which they already have a body of knowledge that competes with the researcher's own discipline in explaining human behavior. Because of this, and because of the social hierarchy of the hospital professions in relation to society as a whole, the researcher cannot enter the mental hospital just by right of having a claim to professional competence in his area. He must either have a special invitation or special permission and this can only be given by the highest social group in the hospital, the psychiatrists. By implication then, the presence of the researcher is only possible by the authority that the psychiatrist has in imposing the researcher on the other professional groups.

The psychiatrist who grants this permission allows, to some extent, the invasion of his own sphere of professional competence and perhaps may even become involved in a challenge to the theoretical structure of his own science.

In the eyes of the other professional groups, the researcher comes as a novice. The mental hospital is not a laboratory where he can work nor is it a primitive group to be lived with. The population and behavior of the hospital is usually unknown to the researcher and he is about to compete with professionals who know more about "their" patients than he could in the short time he is to be there.

Thus, even before he enters the mental hospital the researcher must understand that his very presence, if known to others, will be an imposition on the professional groups already there, that his discipline itself can be considered a threat by others and that his position, in the view of the other professionals, is that of one who can never quite equal their own experience.

\section{Types of Observer Roles in the Mental Hospital}

In following the kinds of observer roles listed by Gold $^{8}$ it will be noted that most of the types named below can be loosely classified either under the Complete Participant or the Observeras-Participant role. Most researchers utilize some kind of interviewing which could come under the Observer-as-Participant role but since the problems in using this technique do not differ markedly from problems in interviewing generally or from problems encountered in the first two types of roles, the interviewing role will not be discussed. Gold's fourth category, the Complete Observer, is rarely encountered in the mental hospital setting since it would require facilities in which the observer could see and hear, but not be seen or heard by either patients or staff.

\section{The Patient Role}

This type of role is assumed when the investigator has himself admitted to the hospital as a patient and lives for a time with other patients, revealing in no way that he is different from them in his function in the hospital social scheme. Several aspects of this role are worth noting:

1. It is usually the role most threatening to other professions. Even if the observer succeeds in concealing his identity to the end of his research, the sudden discovery that one of the patients was a kind of "spy" is extremely upsetting, especially when the professionals have treated this person with an assumption of mental illness. Redlich and Brody ${ }^{9}$ feel that this is an unethical role to assume because it violates the trusting relations between patient and therapist.

2. Because of the barriers, social, emotional, 
and communicative, between professionals and patients (described in greatest detail by Salisbury ${ }^{10}$ ), this role is probably the only means by which to obtain a nearly complete view of patient attitudes and behavior. Caudill ${ }^{11}$ recommends that all persons dealing with mental patients must go through the experience of living on a hospital ward in order to be able to understand the world the patient lives in. Some psychiatrists disagree with this view and feel data collected in a Participant-as-Observer role are more valuable to them, ${ }^{12}$ but this does not imply that this data is necessarily more valuable to the observer.

3. Although most observers do not specifically mention this aspect, the patient role is the most emotionally taxing to the observer. The fact that of all recent investigations reported only Caudill $^{13}$ attempted this role may be some evidence for this fact. Whether the observer is supposed to simulate an illness or "be himself" is not discussed in the literature.

4. The Patient Role is an extremely limited vantage point from which to observe the behavior of any group except patients, and this is further limited to observation of patients on one particular ward unless one has time to observe on several wards over an extended period. Even the observation of attendants who are the occupational group most often in contact with patients does not permit any opportunity for observing attendant social life outside the ward.

In general, the Patient Role is the sine qua non for an observer to take if he wants to fully understand patient behavior, but it is probably the most limited if he wants to study the entire hospital structure. There is strong psychiatric opposition to an observer taking this role in a true participant observer capacity.

\section{The Aide or Attendant Role}

One step above the patient in the hospital social hierarchy ${ }^{14}$ is the aide or attendant. The observer assumes this role simply by taking a job as attendant or aide in a mental hospital. This requires that he wear the uniform of this occupation and perform the duties required while he is observing without his colleagues having knowledge of his intent.

Arnasson ${ }^{15}$ and Gould ${ }^{16}$ were able to compromise the occupational requirements of this role by acting as "floating attendants' assistants." While the attendants' assistant role also linrits the amount of time spent in observation, it permits greater mobility between wards and seems to offer a peculiar vantage point from which to study the morale and behavior of nurses. ${ }^{17}$

Similar to the Patient Role, the Attendant Role restricts the view of other occupational groups. This role, of course, is less demanding emotionally than the Patient Role and does not involve ethical objections. Some salient points are:

1. To follow the Attendant Role strictly limits amount of observation time to the eight hour shift worked.

2. This role prevents obtaining a balanced view of the other professional groups, the patients, and even of other wards.

3. The Attendant Role introduces the element of authority and control over patients. If one wants to study patient behavior this can be an insurmountable barrier and yet it is one that attendants in general seem unaware of. ${ }^{18}$ Salisbury feels that a non-authoritative role is essential in observing any group. ${ }^{19}$

The above two roles are practiced within the classification of the classical definition of participant observation, or under Gold's Complete Participant Type. The roles of nurses, doctors and other professions are not encountered except as informants to observers. For a time, Caudill ${ }^{20}$ assumed the role of an Adjunctive Therapist, but this was in conjunction with other roles so that data on the problems associated with this role are not available, and the possibility of assuming this role for observation remains open.

\section{The Patient's Friend}

Salisbury ${ }^{21}$ assumed this role by telling patients and attendants that he was not a doctor, therapist, patient, or acting in any other of the roles familiar to them. He tried to be of service to staff and patients alike by carrying messages for patients, watching the ward while attendants left, and performing other chores. Chiefly, he impressed upon the patients that he was not part of the social hierarchy nor con- 
cerned for any one patient, but friendly to all. He imposed a new role on the ward structure that allowed him to state his purpose of observation without appearing to observe (he did not take notes on the ward) and yet rendering a kind of service. This kind of role is somewhere between Gold's Complete Participant role and the Participant-as-Observer role, leaning heavily toward the latter.

1. The Patient's Friend role permits observation of patients without the defensive barriers that ordinarily occur between patients and those who have authority over them. It is made clear that the Patient's Friend is without power to make any decisions on patient welfare.

2. Since this role is not part of any occupational group in the hospital, it is a vantage point for studying all groups. The danger of over-identification is minimized.

3. The Patient's Friend has complete freedom of the hospital. He is admitted to staff conferences, can attend residence training courses, and is able to observe interactions between doctors, nurses, and ancillary personnel.

4. Without any specific duties or work schedule the observer can come and go on the ward as he pleases, allowing observation at whatever times he chooses. This also permits short periods of observation after which notes can be taken out of sight of ward personnel.

5. A strain may result from being caught between the attendants and patients in their conflicts. So as not to compromise the role, the Patient's Friend does not reveal information about secret hacksaw blades.

For those who feel comfortable in it, the Patient's Friend role is one that permits a wider view of hospital social life than the two roles previously discussed. In regard to the social structure of the hospital, it is the role that permits least interference with observation while still retaining the pose of doing some useful service.

\section{The Professional Observer Role}

Most observers prefer to drop all pretense of being a member of the hospital structure as it is officially composed or of rendering a service, and seek to impose the new role of Professional Observer upon the social system. Yet, their activities are still frequently labelled "participant observation." This kind of role is more often taken by sociologists than by anthropologists and the cause may be due to a more classical influence in the observer techniques of anthropologists. ${ }^{22}$ The role fits Gold's Participant-as-Observer type.

1. The role of Professional Observer could easily be the most threatening to other professions of all the roles an observer could take. While patients are generally not more threatened by it than other roles, it remains the hardest for all groups to "place" in the social system. It is the role most easily interpreted as being a "spy" for some authority. Thus, an unskilled observer could easily accrue to himself the barriers from all social groups rather than find in this role a bridge across them.

2. By the same token this very aspect of threat can be used by a skillful observer as a research instrument in its own right. This role is not biased by membership in any occupational or professional group and its definitional slackness in the hospital social system permits the role to act as a projective device for individuals in other roles. Anxious attendants will see the observer as someone who is checking up on them. Hopeful patients see him as a new source of information. The way other persons are threatened or made secure by this role tells a great deal about them.

3. The fact that the observer role has no locus or defined position in the social structure permits a maximum of social mobility and contact with all social groups. As a result, the Professional Observer is often used to carry information from one social group to another and this increases the vantage point from which to observe these groups provided the observer is skilled enough to use it to advantage and can incur trust in others.

4. It is the one role in which note-taking is more easily justified in the presence of others. There is no need to explain inappropriate role behavior because the proper business of an observer is to take notes.

5. The Professional Observer role is outside participation in activities more than any other 
role and this can be a good excuse to ask others to explain everything to him. The role does not require that he have knowledge about any other role.

6. This role does not carry with it the requirement of identification with any other role, thereby increasing the objectivity of observation.

In summary, each of the roles an observer can take is peculiar to the hospital social structure. Each role taken within the hospital social structure usually has the disadvantage of limiting observation to the group of which one is a member. This is because in the mental hospital every social group is markedly separate from any other in ways not found in communities. It is not possible for an observer to use participant observation by becoming a member of the society and sampling several roles. In the mental hospital one cannot become a member of the group as a whole; he can only join one of the social subgroups. In this respect the Professional Observer role permits a maximum vantage point for observation of other groups, but it is not an adequate substitute for becoming a member of a community. The Professional Observer role carries with it the penalty of suspicion from other professionals which cannot be easily overcome.

The isolation of the various social groups in the mental hospital cannot be overcome by the use of participant observation in the classical sense or even in the approximation to Gold's Participant-as-Observer. Since a person cannot become a member of the mental hospital subsystem and sample several roles, the observer must either resort to sampling all roles, at separate times, or use a team of observers for each social group in addition to the interview and testing techniques. The marked isolation of the mental hospital social groups is one of the main reasons why a complete ethnography of the mental hospital has never been collected.

A chief theoretical difference between the community and the mental hospital is in the concept of the role of formal member. In the community one can become a formal member by initiation, legal residence, etc. But in the mental hospital no person is in any sense just a member. Every individual can only be linked to the mental hospital by virtue of his professional or patient role with no common denominator of roles in between. Hence, a person who does not clearly belong to one of these roles does not have a right to status in the hospital subsystem. The observer must impose his own role to gain status if he does not participate.

\section{Some Questions About the Use of Other Roles}

In considering the types of observer roles used in the mental hospital, one might ask why more types haven't been utilized. Why not observe from the vantage point of psychiatrist, psychologist or nurse? One of the reasons is that this does not permit maximum contact with patients. Another is that these roles have too much authority over patients and therefore do not allow the minimum of threat necessary for observation. Both reasons are based on the assumption that patients are the chief subjects of observation in the ental hospital. This assumption is based on the cultural belief that mental hospitals exist for the patients.

Another reason for not assuming other professional roles is the technical proficiency and training required before one could safely emulate the required behavior. An observer who wishes to study doctors in the mental hospital cannot go through medical school just to assume a participant role.

In community studies informants make up for the lack of experience the observer has in other roles. In the mental hospital it is difficult for informants to make up for this lack due to the barriers between the professional groups. Salisbury states that he would not have been aware of these barriers if he had taken an attendant's role. ${ }^{23}$ Clearly, before the use of informants can be effective, the observer must have an accurate appraisal of barriers between social groups already in hand.

The concentration on observer roles that allow access to patients has been one of the chief reasons why mental hospital studies are characterized by incompleteness. Even the conscientious attempt of Dunham and Weinberg ${ }^{24}$ to investigate all roles in the mental hospital was criticized by Wilkie ${ }^{25}$ for its incompleteness in covering hospital bureaucracy.

Historically, studies have also tended to incompleteness because of a focusing on small hospitals. The Stanton and Schwartz hypothesis 
At least some aspects of the disturbances of patients are a part of the functioning of the institution 26

is responsible for the launching of many small hospital studies and was itself derived from the study of one such institution. The acceptance of this hypothesis by psychiatrists is the rationale for inviting researchers to study the mental hospital. Salisbury ${ }^{27}$ has criticized this hypothesis on the basis that it was derived from the small hospital where the ratio of staff to patients was very high. In larger hospitals the effect of the staff on the patients is less important than the society that evolves on the ward. But Salisbury uses this as his starting assumption for study and does not follow an etiology of communication that could confirm or refute the hypothesis. His concentration on the ward social system lacks completeness in the same way as the small hospital studies.

\section{Role Conflicts}

The Administrator-Therapist-Researcher Complex

As a result of his training the researcher comes to the mental hospital setting with many biases that conflict directly with the biases of medical training and administrative procedure. Researchers, as a matter of principle, try to keep free from authority in decision making. Academic researchers often do not respect rote memory (a basic method for teaching medicine) or the giving of authoritative opinions. ${ }^{28}$ Yet the stock in trade of the therapist is his opinion, and he uses rote memory to classify illnesses. In addition, ignorance in the therapist's eyes is looked upon as a confession of failure. The researcher does not look upon ignorance as such a threat and readily admits it. In their references to significant units of thought the therapist speaks of a person while the researcher speaks of a variable. The areas of conflict are on four levels:

1. Communication of Knowledge - opinion vs. tentative hypotheses. 2. Methods of Learning - rote vs. learning of concepts. 3. Presentation of Self competent self vs. skeptical self. 4. Units of Thought-getting a person well vs. improving a conceptual system.
The researcher is also confronted with the therapist-administrator paradox in every psychiatrist, a paradox which researchers can avoid by refusing to take responsibility for administrative decisions. As an administrator the psychiatrist of the past (and many in the present) has often been tempted to (1) tighten restrictions in response to crises, (2) use economy as a major goal, (3) treat personnel as deserving. of more privileges than patients, (4) isolate the hospital from the rest of the community, and (5) to "push" patients and personnel to get people well quickly. ${ }^{29}$ As a therapist the psychiatrist wishes to (1) remain non-authoritarian in the face of crises, (2) consider economy second to therapy in importance, (3) feel employees and patients are equal, (4) integrate the hospital with the community, and (5) to let patients progress at their own rate without pressure.

The thought patterns of the therapeutic personnel are often an anathema to the researcher. The mental hospital staff conference is a setting where ad hominem types of argument take precedence over all others, where why a person says what he tes is more important than what he says. 30

In addition to the above complications there is the fact that psychiatrist and researcher are of the same culture but differ greatly in the prestige accruing to their positions. This means that the researcher is not automatically given access to the social life of the psychiatrist, since subtle barriers exist that are not obvious to one who is not already a member of that subgroup. The social status of the psychiatrist both in and out of the hospital setting is a subject not fully explored by hospital studies. Two areas of this conflict are of importance to the observer. One is that the social barriers are likely to prevent accurate observation of the psychiatrists as a group, and the other is that observers who treat patients as equals at the same time they treat psychiatrists as equals must be careful not to incur the enmity of both. ${ }^{31}$

The medical ethic of the therapist does not allow the use of therapy hours for observational studies. 32 Thus, there is no real way to study the relationships between ward life and progress in therapy. This is another of the reasons why mental hospital studies have lacked completeness. It presents a formidable obstacle to study 
of the most important function of the mental hospital.

\section{The Finance vs. Therapy Conflict}

It has already been mentioned that the administrative goals of the psychiatrist often conflict with therapeutic goals in the economic realm. But one of the most neglected areas of study is the social relations between financial bureaucrats of the hospital and the staff and patients. In the last ten years of research reported, only Stein attempts some description of relations between financial-administrative and psychiatric personnel. ${ }^{33}$ Greenblatt, York, and Brown lament the "terrible" influence the financial bureaucrat has, but report little that is functionally useful:

Perhaps one of the most surprising and disconcerting discoveries was the degree of power which could be exercised by persons in charge of the purchase or requisitioning and distribution of supplies for the institution, and by those whose function it was to make repairs and maintain plant and equipment. By their withholding supplies and services ... they could seriously damage staff morale. ${ }^{34}$

Why has the area of finance and maintenance roles been neglected? Probably because of the concentration on the study of patient behavior in the belief that the mental hospital exists for the patients, and possibly because the authority of the psychiatrist in imposing the observer upon other groups does not extend to the financial bureaucracy.

\section{The Political vs. Therapy Conflict}

There is an area of potential social influence not mentioned in hospital studies. It is well known that in many states the attendants are given their jobs through political influence. In effect, this makes the position of superintendent amenable to political pressure if he does not agree to hiring new personnel each time the party in power changes. It also makes many attendants relatively immune to discipline if they are allied with the party in power, while others can become extremely anxious if they are allied with the party out of power. Such a condition affects job security for many and this has ramifications throughout the hospital.

In addition to this, employees are assessed by the party in power for annual contributions. While such a practice is illegal in many states, it goes on informally.

The interference of politics with therapy has never been studied. It is easy to understand why the administrators would not want this fact mentioned in any public report-it might cost them their jobs. In fact, it may well be that studies have not been done in such hospitals because of the fear that political influence might be discovered.

The areas of role conflict are financial, political, therapeutic, and administrative. The researcher will conflict with professionals in each of these roles because his aims and training contradict in many ways the aims of the finance officer, therapist, administrator, or other persons with whom he comes in contact. The mental hospital subsystem is not an open group to the researcher. He cannot collect his data at random but must work in the areas that are least threatening to the hospital authorities.

\section{$A$ Look at the Future}

With the passage of the Community Mental Health Centers Act of 1963 (Public Law 88-164) a new era has begun regarding the treatment of the mentally ill. This bill provides an initial sum of 270 million dollars for the construction of community mental health center buildings throughout the nation. But more than this single bill is involved. The entire trend in psychiatric care is a movement toward the small (200 bed) community mental hospital or community medical center with a mental unit. ${ }^{35}$

The future of the mental hospital as it now exists remains uncertain, and how it will be related to the new outcrop of community centers has yet to be defined. But the placement of these centers within the community poses an even larger problem. It opens up a new area to the applied social scientists who are specialized in both community and mental health studies. Mental health authorities are not only concerned with the placement of these buildings in the community but with the psychological preparation of the community for the new institution. Mental health education of the community is not an easy task and requires skilled behavioral scientists. ${ }^{36}$ 
There is a need to know areas of possible resistance to this program, social structures and hierarchies that must be approached to ease the transition, and attitudes and beliefs that must be overcome before such community centers are possible.

Partly as a result of past studies in the mental hospital it has been definitely shown that the relative isolation of the mental hospital from the community hindered therapy. ${ }^{37}$ This fact has been influential in the decision to reintegrate the mental hospital into the community.

But what of the large number of mental hospitals remaining? It is not likely that they will cease to function in the immediate future. New programs in day hospitals, out-patient treatment, and team-therapy are awaiting investigation in these hospitals. Because of the isolation and lack of communication between social groups in the mental hospital, Caudill proposes that a clinical anthropologist be added to the permanent staff of the mental hospital to coordinate the social groups. ${ }^{38}$ There is no reason why any skilled behavioral scientist could not act in the same role, but such a position may not be necessary if the team plan is effective. The team plan consists of having a psychiatrist, psychologist, nurse, social worker, and attendant operate together on a therapy program for a given number of patients. Atpresent a study is being planned that would evaluate whether the team plan in conjunction with a campus plan of hospital buildings (where each team is housed in a separate building) is effective in increasing interaction and communication between the social groups that were previously found to be so isolated. ${ }^{39}$

The work of the researcher who uses observational techniques in the mental hospital will increase in the future because of the expansion of the mental hospital into the community and the acceleration of new therapeutic programs. It is hoped that with the consideration of the unique qualities found in the mental hospital, future researchers will operate in groups that will provide a comprehensive coverage of social life in the hospital and yield a complete picture of the mental hospital as a subsystem of our society.

\section{Conclusions}

The use of participant observation in the classical sense has been found to be of limited value in the mental hospital. A participant observer in the role of a patient is considered unethical and a participant observer in the attendant's role has a very limited vantage point for observation. The use of other roles in the hospital social structure is even more difficult. A Professional Observer role which does not involve much if any participant observation, but which allows observation as a known investigator, of all roles has been used with greatest success in the past.

For a comprehensive study of the mental hospital, however, a team of observers is recommended'with an observer at each social level coordinated by an observer who acts in the Professional Observer role.

Some aspects of the mental hospital act to hamper observation. The isolation of the social groups permits only inadequate observation across groups when an observer identifies with any one group. Assumptions of the researcher act to hamper his effectiveness with medical staff who have disciplines sometimes at variance with research values and who often express themselves in different modes. The roles of administrators, finance officers, therapists, and others conflict with the observer role to the point of excluding observation from certain critical areas of study. Since the field of observational study in the mental hospital seems to have an expanding potential, it seems imperative to realize that the participant observation techniques of community studies cannot be transferred to the mental hospital without extensive modification in order to prevent repetition of past mistakes.

\footnotetext{
${ }^{1}$ Florence Kluckhohn, "The Participant Observer Technique in Small Communities," American Journal of Sociology, XLVI (November, 1940), 331.

${ }^{2}$ Nicholas Babchuk, "The Role of the Researcher as Participant Observer and Participant-as-Observer in the Field Situation," Human Organization, XXI (Fall, 1962), 225.

${ }^{3}$ Raymond L. Gold, "Roles in Sociological Field Observations," Social Forces, XXXVI (1958), 217-223.

${ }^{4}$ Parsons says that the mental hospital is not a community, kinship group, or an association; it is a subsystem of a society. Talcott Parsons, "The Mental Hospital as a Type of Organization," in Milton Greenblatt, Daniel Levinson, and Richard Williams (eds.), The Patient and the Mental Hospital, Free Press, Glencoe, Ill., 1957, 108-129.
} 
${ }^{5}$ Esther Brown, H. Warren Dunham, and Richard York, "The Application of the Sciences of Social Behavior in Ward Settings," in Milton Greenblatt, et al., ibid., 479-498.

${ }^{6}$ Daniel Levinson, "The Mental Hospital as a Research Setting: A Critical Appraisal," in Milton Greenblatt, of al., ibid., 634.

${ }^{7}$ Refer back to Gold's classification, page 166 .

8 Ibid., page 166.

${ }^{9}$ F. C. Redlich and E. B. Brody, "Emotional Problems of Interdisciplinary Research in Psychiatry," Psychiatry, XVIII, 1955, 233-239.

${ }^{10}$ Richard Salisbury, Structures of Custodial Care, University of California Press, Berkeley, 1962.

11 William Caudill, The Psychiatric Hospital as a Small Society, Harvard University Press, Cambridge, 1958.

${ }^{12}$ See Redlich's introduction to Caudill, ibid., ix.

${ }^{13}$ Caudill, ibid.

${ }^{14}$ See Martin Loeb, "Role Definition in the Social World of a Psychiatric Hospital," in Milton Greenblatt, et al., op. cit., 14-19, for a description of formal and informal social hierarchies in the mental hospital.

${ }^{15} \mathrm{~B}$. B. Arnasson, "Care and Cure as Functions of a State Mental Hospital," unpublished Ph.D. Dissertation, Radcliffe College, 1958.

${ }^{16} \mathrm{~N}$. Gould, "Problems of Organizational Change in Public Mental Hospitals," unpublished Ph.D. Dissertation, Dept. of Social Relations, Harvard University, 1960.

${ }^{17}$ Salisbury, op. cit., 14.

${ }^{18}$ Salisbury, ibid.

${ }^{19}$ Salisbury, ibid.

20 Caudill, op. cit., 23.

${ }^{21}$ Salisbury, op. cit., 3-10.

22 B. Paul, "Interview Techniques and Field Relationships," in Alfred Kroeber (ed.), Anthropology Today, University of Chicago Press, Chicago, 1953.

${ }^{23}$ Salisbury, op. cit., 14.
${ }^{24} \mathrm{H}$. Warren Dunham and S. Kirson Weinberg, The Culture of the State Mental Hospital, Wayne State University Press, Detroit, 1960.

${ }^{25} \mathrm{R}$. Wilkie, book review on The Culture of the State Mental Hospital, American Anthropologist, LVIII, 1961, 664-665.

${ }^{26}$ Alfred Stanton and Morris Schwartz, The Mental Hospital, Basic Books, New York, 1954, 12.

27 Salisbury, op. cit.

${ }^{28}$ E. Galioni, R. Notman, A. Stanton, and R. Williams, "The Nature and Purposes of Mental Hospital Wards," in Milton Greenblatt, et al., op. cit., 327-356.

${ }^{29}$ Milton Greenblatt, "The Psychiatrist as Social System Clinician," in Milton Greenblatt, et a1., ibid., 317 323.

${ }^{30}$ Stanton and Schwartz, op. cit., 18.

${ }^{31}$ Dunham and Weinberg, op. cit., 11-12.

32 Caudill, op. cit., 5.

${ }^{33}$ William Stein, "Patterns of a Peruvian Mental Hospital," The International Journal of Social Psychiatry, IX, 1963, No. 3, 208-215.

${ }^{34}$ Milton Greenblatt, Richard York and Esther Brown, From Custodial to Therapeutic Patient Care in Mental Hospitals, Russell Sage Foundation, 1955, 20.

${ }^{35}$ Dunham and Weinberg, op. cit.

${ }^{36}$ See John and Elaine Cumming, "Mental Health Education in a Canadian Community," in Benjamin Paul (ed.), Health, Culture and Community, Russell Sage, New York, 1955, 43-70; and Kaspar Naegle, "A Mental Health Project in a Boston Suburb," in the same book, 295-321.

37 John and Elaine Cumming, "Social Equilibrium and Social Change in the Large Mental Hospital," in Milton Greenblatt, et al., op. cit., 49-71.

${ }^{38}$ Caudill, op. cit.

39 The Woodsview project under the Architectural Environment and Human Behavior Program at Topeka State Hospital. 